\title{
MUCH DOES NOT HELP MUCH: 3D PARETO FRONT OF SAFETY, COMFORT AND ENERGY CONSUMPTION FOR AN ACTIVE PNEUMATIC SUSPENSION STRUT
}

\author{
Manuel Rexer, Nicolas Brötz, Peter F. Pelz* \\ Institut für Fluidsystemtechnik, Technische Universität Darmstadt, Otto-Berndt-Straße 2, 64287 Darmstadt \\ * Corresponding author: Tel.: +49 6151 1627100; E-mail address: peter.pelz@fst.tu-darmstadt.de
}

\begin{abstract}
With regard to autonomous driving the demands on comfort are increasing. This makes it attractive to use active suspension systems. The system developed at TU Darmstadt is able to increase driving comfort up to $28 \%$ while maintaining driving safety compared to a passive suspension system.

This paper investigates the influence of available energy and power of the active system. The investigation is based on a simulation of a quarter car model and an uneven country road. This paper shows that the more energy the active system has at its disposition, the greater is the range between a comfortable and a sporty chassis. Furthermore the driving comfort can be increased by $28 \%$ with constant driving safety. The average power required for this is $15 \mathrm{~W}$ and the maximum power is $300 \mathrm{~W}$.
\end{abstract}

Keywords: air spring, acitve suspension, energy demand, power demand

\section{INTRODUCTION}

With regard to autonomous driving the demands on comfort are increasing. Passengers may engage in other activities during the ride but may also suffer from kinetosis more frequently $[1,2]$. This makes it attractive to use active systems in the suspension system. Previous studies showed that an active air suspension system as in Figure 1 is well suited to calm the chassis and increase driving comfort [3-5]. The system developed within the Collaborative Research Center 805 "Control of uncertainty in load carrying structures in mechanical engineering", increases driving comfort by up to $28 \%$ while maintaining driving safety compared to a passive air suspension [6]. These results were found in simulations as well as in hardware-in-the-loop experiments $[5,6]$.

The average available actuating power is one influencing factor to increase driving safety and comfort. The average available actuating power represents the energy demand of the active system which makes it an important indicator as especially for electrified vehicles energy supply is limited. This is simulatively investigated on the basis of the vertical dynamics of a quarter car model. Therefore, in the following first is the quarter car model as well as the principle of the active air spring is introduced. Then the performance parameters and the calculation of the actuator power are presented and finally results are discussed.

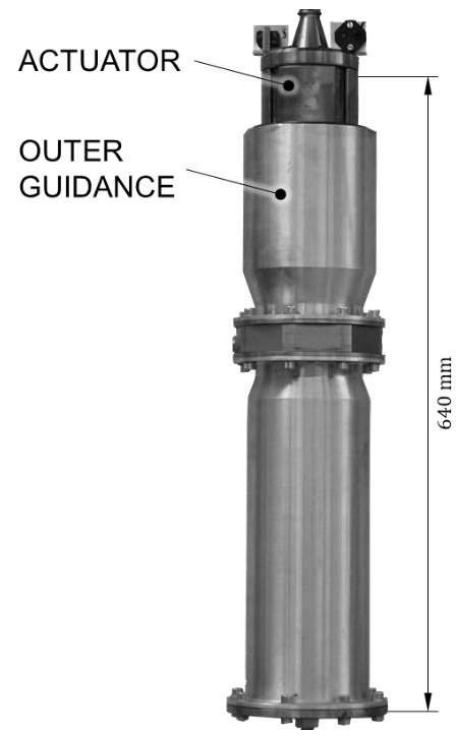

Figure 1: Active air spring. 


\section{METHOD}

\section{1. system description}

\section{quarter car model}

The actuator of the active air spring is considered in the quarter car model as a force connected in parallel both to spring and damper (c.f. Figure 2).

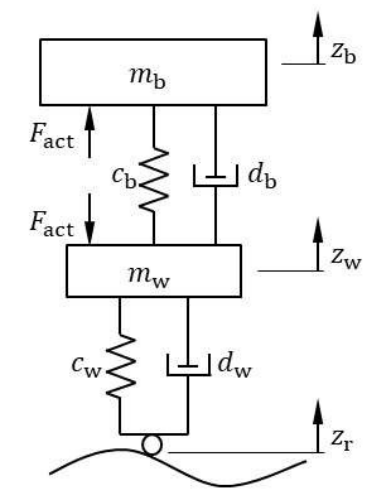

Figure 2: Quarter car model with active suspension system.

The equations of motion of this quarter car are

$m_{\mathrm{b}} \ddot{z}_{\mathrm{b}}+d_{\mathrm{b}} \dot{z}+c_{\mathrm{b}} \mathrm{z}+F_{\mathrm{act}}=0$,

$m_{\mathrm{w}} \ddot{z}_{\mathrm{w}}+d_{\mathrm{w}}\left(\dot{z}_{\mathrm{w}}-\dot{z}_{\mathrm{r}}\right)+c_{\mathrm{w}}\left(z_{\mathrm{w}}-z_{\mathrm{r}}\right)-d_{\mathrm{b}} \dot{z}-$

$c_{\mathrm{b}} \mathrm{z}-F_{\mathrm{act}}=0$,

with the deflection $z$ and the deflection velocity $\dot{z}$

$z=z_{\mathrm{b}}-z_{\mathrm{w}}$

$\dot{z}=\dot{z}_{\mathrm{b}}-\dot{z}_{\mathrm{w}}$.

All results refer to a quarter car model with parameters based on a middle class car as in Table 1.

Table 1: Parameters of the quarter car model.

\begin{tabular}{clcl}
\hline$m_{\mathrm{b}}$ & $270 \mathrm{~kg}$ & $m_{\mathrm{w}}$ & $50 \mathrm{~kg}$ \\
$c_{\mathrm{b}}$ & $10 \mathrm{~N} / \mathrm{mm}$ & $c_{\mathrm{w}}$ & $200 \mathrm{~N} / \mathrm{mm}$ \\
$d_{\mathrm{b}}$ & $1140 \mathrm{Ns} / \mathrm{m}$ & $d_{\mathrm{w}}$ & $566 \mathrm{Ns} / \mathrm{m}$ \\
\hline
\end{tabular}

\section{active air spring}

This paper investigates an active air spring suspension system. The force of an active air spring is given by a static load $F_{0}$, a deflection force $c z$ and the actuator force

$F=F_{0}+c z+F_{\text {act }}[6]$.

In principle the actuator force is changeable either by (i) the air mass, (ii) the load carrying area or (iii) the volume of the air spring. Hedrich [6] shows that only a change of the load carrying area is useful.

An air spring is usually sealed by rolling bellows which are guided on the inside by a piston and on the outside by an outer guidance. However, the load carrying area of an air spring is approximately

$A_{\mathrm{c}} \approx \pi\left(\frac{r_{\mathrm{o}}+r_{\mathrm{p}}}{2}\right)^{2}$.

The load carrying area at the active air spring varies by changing the piston radius $r_{\mathrm{p}}$ (c.f. Figure 3) [3]. For this purpose segments are moved as hydraulically single-acting cylinders. The return takes place through the bellows and the pressure in the air spring.

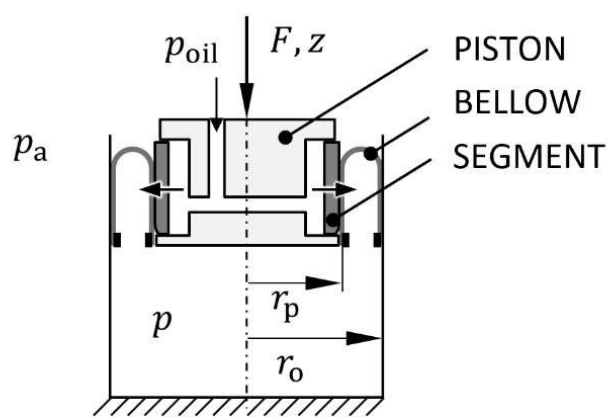

Figure 3: Principle of the active air spring. Singleacting cylinder that changes the load carrying area.

Whenever the actuator force increases, oil must be forced into the actuator. A pump and valve supply the hydraulic power. To reduce the actuator force, the oil is drained.

Measurements show approximately a PT1 transfer characteristic for the actuator with a cutoff frequency of $5 \mathrm{~Hz}$ and a maximum actuating force of $F_{\text {act,max }}= \pm 1 \mathrm{kN}$ [7].

\section{performance indicators}

In order to evaluate the performance of the actuator, driving safety and driving comfort are taken into account. Mitschke shows the according definitions [8]. The standard deviation of the relative wheel load variation is regarded as driving safety

$\frac{\sigma\left(\mathrm{F}_{\mathrm{W}}\right)}{F_{\mathrm{W}, 0}}=\frac{1}{F_{\mathrm{W}, 0}} \sqrt{\frac{1}{T} \int_{0}^{T}\left(F_{\mathrm{W}}(t)\right)^{2} \mathrm{~d} t}$.

The standard deviation of the body acceleration is regarded as driving comfort 
$\sigma\left(\ddot{z}_{\mathrm{b}}\right)=\sqrt{\frac{1}{T} \int_{0}^{T}\left(\ddot{z}_{\mathrm{b}}(t)\right)^{2} \mathrm{~d} t}$.

Both performance indicators are determined without frequency weights. The results depend on 1. the road,

2. the driving speed and

3 . the car.

This investigation excites the system applying a stochastically generated road. The road corresponds to an uneven country road at a speed of $70 \mathrm{~km} / \mathrm{h}$ and can be generated from white noise $n$ by form filters [6]. The parameters of the country road are from Mitschke [8] with a waviness of $w=2$ and an unevenness dimension of $\Phi_{\mathrm{z}_{\mathrm{r}} z_{r}, \Omega}(1 / \mathrm{m})=\pi \cdot 12.2 \cdot 10^{-6} \mathrm{~m}^{3}$.

Figure 4 shows the active and passive boundary line next to the passive reference car in a conflict diagram. The reference car results from the simulation of the quarter car with parameters from Table 2 and no actuator force $F_{\text {act }}=0$.

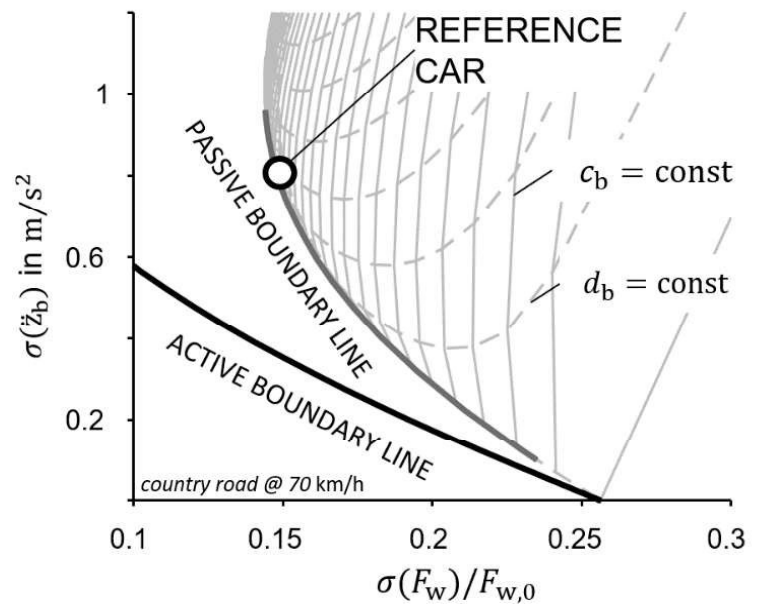

Figure 4: Conflict diagram for driving comfort and safety.

The passive boundary line is reached by varying stiffness and damping. The isolines for $c_{b}=$ const or $d_{\mathrm{b}}=$ const show this as well. The active boundary line assumes an ideal actuator with the transfer function of 1 and a maximum actuating force $F \rightarrow \infty$. Active suspension systems can therefore cross the passive boundary line.

\section{controller design}

The controller for the active system is designed with a model-based H2-optimal controller design according to Lenz et al. [7]. For this purpose the objective function considers driving safety and driving comfort. Since they are in conflict with each other, a compromise between the two can be found by weighting them. A pareto front represents the results in the conflict diagram as in Hedrich [6] (see Figure 4).

\section{2. power demand}

\section{average power demand}

The power of the actuator is given by the product of deflection velocity and the actuator force

$P(t)_{\text {act }}=\dot{z}(t) F_{\text {act }}(t)$.

Due to the actuator's functionality power at pressure $P(t)_{\text {act,p }}$ meaning increasing actuator forces and at tension $P(t)_{\text {act,t }}$ meaning decreasing actuator forces need to be distinguished. The pressure power is determined by

$P(t)_{\mathrm{act}, \mathrm{p}}=\left\{\begin{array}{ll}0 & \forall \frac{\mathrm{d} F_{\mathrm{act}}(t)}{\mathrm{d} t} \leq 0 \\ \left|P(t)_{\mathrm{act}}\right| & \forall \frac{\mathrm{d} F_{\mathrm{act}}(t)}{\mathrm{d} t}>0\end{array}\right.$,

and tension power by

$P(t)_{\mathrm{act}, \mathrm{t}}=\left\{\begin{array}{ll}\left|P(t)_{\mathrm{act}}\right| & \forall \frac{\mathrm{d} F_{\mathrm{act}}(t)}{\mathrm{d} t} \leq 0 \\ 0 & \forall \frac{\mathrm{d} F_{\mathrm{act}}(t)}{\mathrm{d} t}>0\end{array}\right.$.

External energy sources supply the pressure power.

Due to the fact that the resetting forces of the air spring cause the decrease of the actuator force, external power supply does not provide the tension power.

The power demand of an active suspension system is considered as an average power demand, which is equivalent to the required energy. The average power is calculated over a time average of

$$
\bar{P}=\frac{1}{T} \int_{0}^{T} P(t) \mathrm{d} t .
$$

The average power demand of the active system can therefore be specified as

$\bar{P}_{\text {act }}=\bar{P}_{\text {act }, \mathrm{p}}-\gamma \bar{P}_{\text {act }, \mathrm{t}}$.

$\gamma$ is the energy recuperation factor, which varies between 0 (no energy recuperation) and 1 (total energy recuperation). This investigation does not take energy recuperation into account as a conservative estimation $(\gamma=0)$.

The energy required by the active system is obtained from the product of average power $\bar{P}$ and duration $T$. 


\section{consideration of the available power in the} controller design

Due to nonlinearity the actuator power is simulated in time domain in contrast to controller design simulated in frequency domain.

Therefore Figure $\mathbf{5}$ shows the extended controller design process.

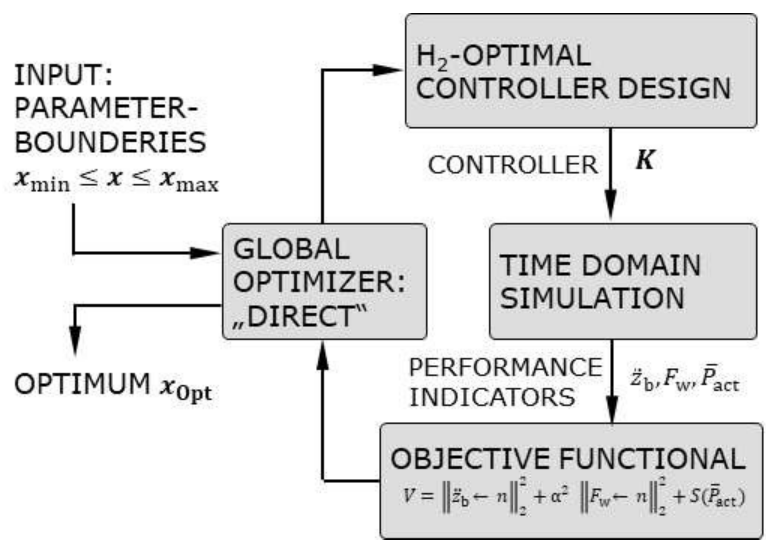

Figure 5: Extended controller design to consider the available actuator power (in accordance to [6]).

The global optimizer "DIRECT" [9] is used in addition to controller optimization. In order to influence the power of the actuator the parameters of the transfer function of the actuator are adjusted. Table 2 presents the parameter limits.

Table 2: parameter range of global optimization.

\begin{tabular}{ll}
\hline parameter & Range \\
\hline maximum force $F_{\mathrm{PT} 1}$ & $0 \ldots 1 \mathrm{kN}$ \\
base frequency $f_{\mathrm{PT} 1}$ & $0 \ldots 30 \mathrm{~Hz}$ \\
\hline
\end{tabular}

The global optimization specifies a transfer function for the actuator determining the optimal controller. The model is then simulated in time domain to determine the evaluation variables for the objective function

$\min \left\|\ddot{z}_{\mathrm{b}} \leftarrow n\right\|_{2}^{2}+\alpha^{2}\left\|F_{\mathrm{w}} \leftarrow n\right\|_{2}^{2}+$ $S\left(\bar{P}_{\text {act }}\right)$.

A Big-M method takes compliance with the actuator power into account

$S\left(\bar{P}_{\mathrm{act}}\right)= \begin{cases}0 & \forall \bar{P}_{\mathrm{act}} \leq \bar{P}_{\mathrm{act}, \mathrm{max}} . \\ M & \forall \bar{P}_{\mathrm{act}}>\bar{P}_{\mathrm{act}, \mathrm{max}}\end{cases}$

The used optimizer "DIRECT" searches for the optimum in both depth and width but cannot guarantee to find the global optimum. This may lead to inaccuracies in the results which is not further examined in this paper.
These basic investigations take no efficiency of the actuator into account. Dietrich et al. [10] considers the efficiency of the example actuator.

\section{RESULTS}

\subsection{D pareto front}

Figure 6 shows the result in a 3D Pareto front where the driving comfort and driving safety are plotted against the average available actuating power. This corresponds to the energy requirement of the system. Inconsistencies as kinks and discontinuities in the lines occur from the optimizer not reaching the global optimum. These are neglected, as the fundamental trends are nevertheless recognizable and not influenced by them.

Starting from the passive reference car which has no power available the active chassis enables increasing flexibility in the weighting between driving comfort and safety as the available power increases. The driver has the choice between a comfortable and a sporty chassis.

In total the ideally active system requires around $90 \mathrm{~W}$ of average power i.e. $360 \mathrm{~W}$ for the whole car.

In addition a saturation occurs. With a power of about $50 \mathrm{~W}$ the driving safety cannot be increased by further available actuating power. It is only possible to increase maximum comfort. This can be seen from the fact that the upper end of the curves do not drop increasing the power in terms of driving safety, but the lower end of the curve drops further in terms of driving comfort.

\section{2. much does not help much: average power demand}

Considering driving comfort of the reference car with a constant safety level of $\sigma\left(F_{\mathrm{w}}\right) / F_{\mathrm{w}, 0}=0.149$, Figure 7 is obtained.

The driving comfort increases with increasing available average actuating power. However, saturation occurs. For this vehicle driving comfort improves by about $28 \%$. This value is 


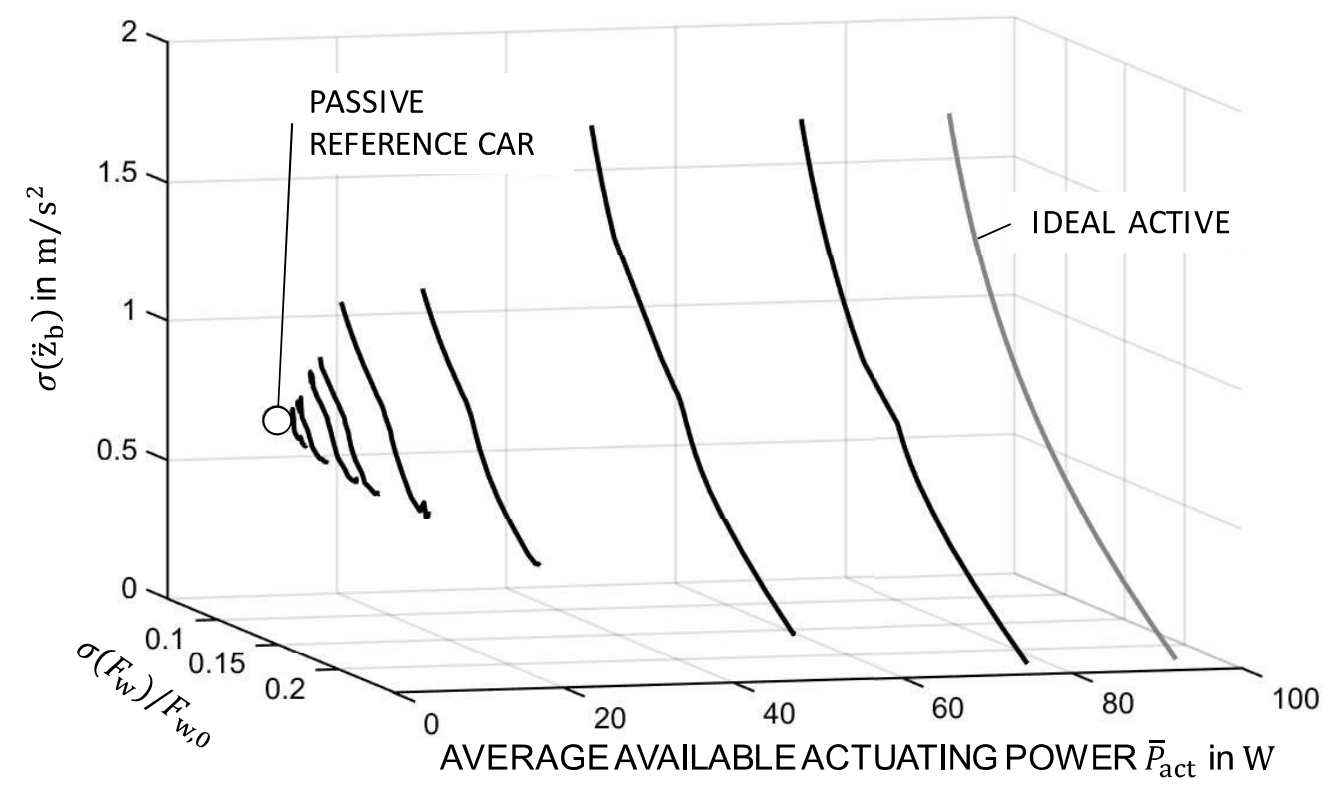

Figure 6: $3 \mathrm{D}$ pareto front as a function of the average available actuating power.

already reached for an average available actuating power of $15 \mathrm{~W}$.

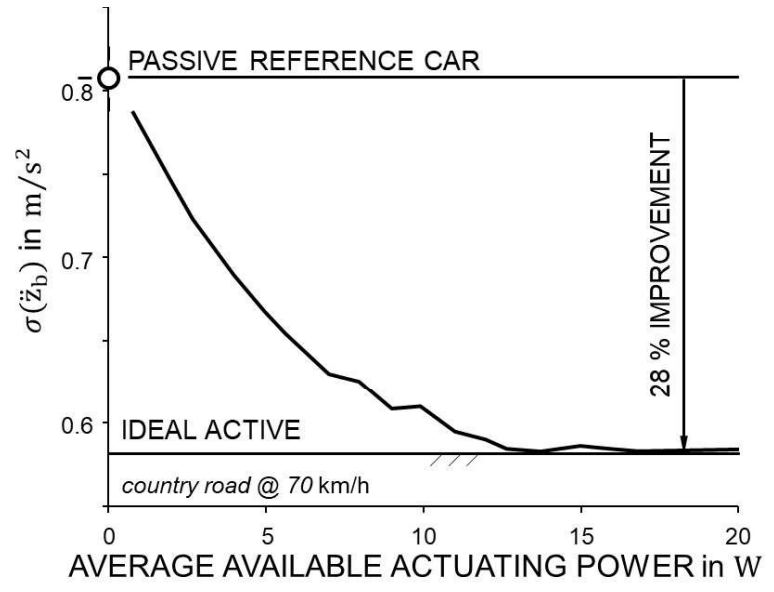

Figure 7: Driving comfort as a function of the average available actuating power with a constant driving safety.

\section{3. peak power demand}

Finally Figure 8 investigates the peak power demand of the active suspension system and considers both the maximum occurring power and the 0.9974 quantile. The second value therefore takes $99.74 \%$ of the power occurring in the simulation into account. However the statistical value is more significant since deviations between simulation and reality do not have an effect as significant as the maximum power.

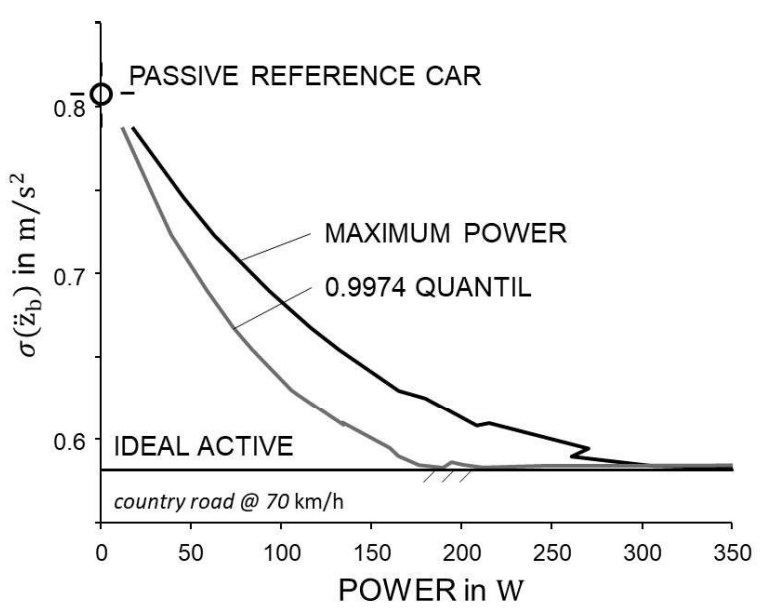

Figure 8: Driving comfort as a function of the maximum power and the 0.9974 Quantil power with a constant driving safety.

For the peak power demand, a similar picture is obtained as for the average available power demand. If starting from the passive reference car the driving safety is kept constant and only the driving comfort is improved. The maximum power requirement is about $300 \mathrm{~W}$, the 0.9974 quantile is saturated at $180 \mathrm{~W}$. The peak powers are therefore at least one order of magnitude greater than the average powers. 


\section{CONCLUSION}

The available power has a considerable influence on the performance of the system. Thus the driver with more available power can choose a larger range between driving safety and driving comfort.

Furthermore to increase driving comfort while maintaining driving safety saturation occurs and only $15 \mathrm{~W}$ of average power is required to achieve an improvement of $28 \%$. This circumstance is advantageous for autonomous driving electrified vehicles as the energy supply is limited there. Also the maximum power saturates at a level of $300 \mathrm{~W}$.

\section{ACKNOWLEDGMENT}

Funded by the Deutsche Forschungsgemeinschaft (DFG, German Research Foundation) - project number 57157498 - SFB805.

\section{NOMENCLATURE}

$A_{\mathrm{c}} \quad$ load carrying area

$c_{\mathrm{b}} \quad$ body stiffness

$c_{\mathrm{w}} \quad$ wheel stiffness

$d_{\mathrm{b}} \quad$ body damping

$d_{\mathrm{w}} \quad$ wheel damping

$F_{\text {act }}$ actuator force

$F \quad$ air spring force

$F_{\mathrm{w}} \quad$ wheel load

$F_{\mathrm{W}, 0} \quad$ static wheel load

$K \quad$ controller

$m_{\mathrm{b}} \quad$ body mass

$m_{\mathrm{w}} \quad$ wheel mass

$n \quad$ white noise

$P \quad$ power

$p \quad$ air spring pressure

$p_{\mathrm{a}} \quad$ ambient pressure

$p_{\text {oil }}$ oil pressure

$\bar{P} \quad$ average power

$P_{\text {act }}$ actuator power

$P_{\text {act, }}$ actuator pressure power

$P_{\text {act, } \mathrm{t}}$ actuator tension power

$r_{\mathrm{o}} \quad$ outer guidance radius

$r_{\mathrm{p}} \quad$ piston radius

$S$ penalty

$t$ time

$T$ duration

$w \quad$ waviness

$z \quad$ deflection

$\dot{z} \quad$ deflection velocity

$\begin{array}{ll}Z_{\mathrm{b}} & \text { body excitation } \\ \dot{Z}_{\mathrm{b}} & \text { body velocity } \\ \ddot{Z}_{\mathrm{b}} & \text { body acceleration } \\ Z_{\mathrm{w}} & \text { wheel excitation } \\ \dot{Z}_{\mathrm{w}} & \text { wheel velocity } \\ \ddot{Z}_{\mathrm{w}} & \text { wheel acceleration } \\ z_{r} & \text { road excitation } \\ \alpha & \text { weighting factor } \\ \gamma & \text { energy recuperation factor } \\ \sigma & \text { standard deviation } \\ \Phi_{z_{r} z_{r}, \Omega} & \text { unevenness dimension }\end{array}$

\section{REFERENCES}

[1] Heinrichs D (2015) Autonomes Fahren und Stadtstruktur. In: Maurer M, Gerdes JC, Lenz B, Winner H (Hrsg) Autonomes Fahren: Technische, rechtliche und gesellschaftliche Aspekte. Springer Berlin Heidelberg, Berlin, Heidelberg, S 219-239

[2] Sivak M, Schoettle B (2015) Motion Sickness in Self-Driving Vehicles

[3] Hedrich P, Johe M, Pelz PF (2017) Aktor mit einem linear verlagerbaren Stellglied (DE 102015120011 A1)

[4] Hedrich P, Lenz E, Brötz N, Pelz PF (Hrsg) (2018) Active Pneumatic Suspension for Future Autonomous Vehicles: Design, Prove of Concept and Hardware-in-the-Loop Simulations, Bd 3

[5] Hedrich P, Lenz E, Pelz PF (2018) Minimizing of Kinetosis during Autonomous Driving. ATZ Woldwide 120(7-8):68-75

[6] Hedrich P (2018) Konzeptvalidierung einer aktiven Luftfederung im Kontext autonomer Fahrzeuge, 1. Aufl. Forschungsberichte zur Fluidsystemtechnik, Bd 20. Shaker

[7] Lenz E, Hedrich P, Pelz PF (2018) Aktive Luftfederung - Modellierung, Regelung und Hardware-in-the-Loop-Experimente. Forschung in Ingenieurwesen:1-15. doi:10.1007/s10010018-0272-2

[8] Mitschke M, Wallentowitz H (2014) Dynamik der Kraftfahrzeuge, 5. Aufl, Wiesbaden

[9] Finkel DE (2003) DIRECT Optimization Algorithm User Guide

[10] Dietrich I, Hedrich P, Bölling C, Brötz N, Geßner F, Pelz PF (2018) Concept of a Resilient Process Chain to Control Uncertainty of a Hydraulic Actuator. AMM 885:156-169. doi:10.4028/www.scientific.net/AMM.885.156 